\section{BRAZIULIAN JOURNAL \\ OF MEDICAL AND BIOLOGICAL RESEARCH}

www.bjournal.com.br
ISSN 1414-431X

Volume 45 (12) 1102-1340 December 2012

\section{BIOMIDICAL SCIENCES}

AND

CLINICAL INVESTIGATION

Braz J Med Biol Res, December 2012, Volume 45(12) 1327-1333

doi: 10.1590/S0100-879X2012007500140

p16 (INK4a) has clinicopathological and prognostic impact on oropharynx and larynx squamous cell carcinoma

S.D. Silva, S. Nonogaki, F.A. Soares and L.P. Kowalski

The Brazilian Journal of Medical and Biological Research is partially financed by

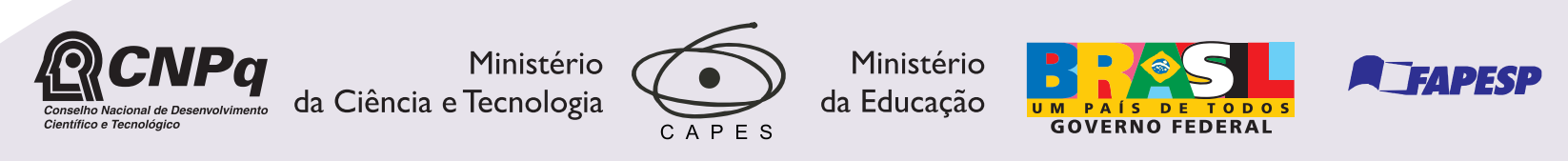

Institutional Sponsors

Sciefo
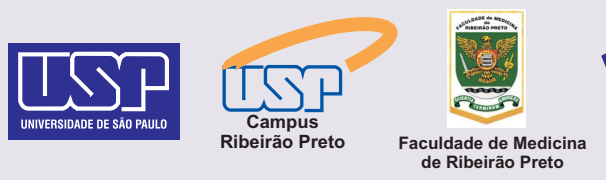

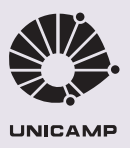

UNICAMP $\oplus$ SHIMADZU

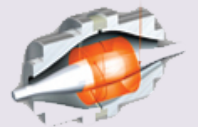

1D Associaçäo Explore High - Performance MS Orbitrap Technology In Proteomics \& Metabolomics analitica $\underset{\text { analiticaweb.com.br }}{\text { Thermo }}$ S I N TIFIC 


\title{
p16 (INK4a) has clinicopathological and prognostic impact on oropharynx and larynx squamous cell carcinoma
}

\author{
S.D. Silva ${ }^{1,2,3}$, S. Nonogaki ${ }^{4}$, F.A. Soares ${ }^{4,5}$ and L.P. Kowalski ${ }^{1}$ \\ 1Departamento de Cirurgia de Cabeça e Pescoço e Otorrinolaringologia, \\ Hospital A.C. Camargo, São Paulo, SP, Brasil \\ 2Department of Oncology, Lady Davis Institute for Medical Research and Segal Cancer Centre, \\ Sir Mortimer B. Davis-Jewish General Hospital, McGill University, Montreal, Quebec, Canada \\ ${ }^{3}$ Department of Otolaryngology-Head and Neck Surgery, Jewish General Hospital, McGill University, \\ Montreal, Quebec, Canada \\ ${ }^{4}$ Departamento de Anatomia Patológica, Hospital A.C. Camargo, São Paulo, SP, Brasil \\ ${ }^{5}$ Departamento de Estomatologia, Faculdade de Odontologia, Universidade de São Paulo, São Paulo, SP, Brasil
}

\begin{abstract}
CDKN2A encodes proteins such as p16 (INK4a), which negatively regulate the cell-cycle. Molecular genetic studies have revealed that deletions in CDKN2A occur frequently in cancer. Although p16 (INK4a) may be involved in tumor progression, the clinical impact and prognostic implications in head and neck squamous cell carcinoma (HNSCC) are controversial. The objective of this study was to evaluate the frequency of the immunohistochemical expression of p16 (INK4a) in 40 oropharynx and 35 larynx from HNSCC patients treated in a single institution and followed-up at least for 10 years in order to explore potential associations with clinicopathological outcomes and prognostic implications. Forty cases $(53.3 \%)$ were positive for p16 (INK4a) and this expression was more intense in non-smoking patients $(P=0.050)$, whose tumors showed negative vascular embolization $(P=0.018)$, negative lymphatic permeation $(P=0.002)$, and clear surgical margins $(P=0.050)$. Importantly, on the basis of negative p16 (INK4a) expression, it was possible to predict a probability of lower survival $(P=0.055)$ as well as tumors presenting lymph node metastasis $(P=$ 0.050) and capsular rupture $(P=0.0010)$. Furthermore, increased risk of recurrence was observed in tumors presenting capsular rupture $(P=0.0083)$. Taken together, the alteration in $\mathrm{p} 16(\mathrm{INK} 4 \mathrm{a})$ appears to be a common event in patients with oropharynx and larynx squamous cell carcinoma and the negative expression of this protein correlated with poor prognosis.
\end{abstract}

Key words: Head and neck cancer; Squamous cell carcinoma; Prognostic value; p16

\section{Introduction}

CDKN2A (also known as ARF, MLM, p14, p16, p19, CMM2, INK4, MTS1, TP16, CDK4I, CDKN2, INK4a, p14ARF, p19Arf, p16INK4, p16INK4a, and CDKN2A), a tumor suppressor gene mapped on chromosome 9p21, encodes proteins, whose main function is the negative cell-cycle regulation (1). The p16 (INK4a) protein is a cyclindependent kinase inhibitor $(\mathrm{CDKI})$ that reduces the rate of the cell-cycle by inactivation of specific cyclin-protein kinase complexes such as cyclin-dependent kinases (CDK) type D1 (CCND1), CDK4, and CDK6 (2-4). The CDKs phosphorylate the retinoblastoma protein $(p R b)$, which results in a conformational change leading to the interruption of the cell-cycle progression $(5,6)$. The inactivation of either p16 (INK4a) or pRb function allows the cell to enter in the $S$ phase after only a pause at the G1 checkpoint (6).

The loss of p16 (INK4a) through homologous deletion, point mutation or methylation-induced promoter silencing has also been widely observed in cancer cell lines and some malignant tumors, such as acute lymphoblastic leukemia $(3,4,7)$, melanomas (8), pancreatic cancer (9), esophagus (10), lung (11), bladder (1), cervical (12), and head and neck squamous cell carcinomas (HNSCC) $(6,13)$. Additionally, the absence of p16 (INK4a) is an early and often critical event in tumor progression, once that deletion in at least one copy is quite high in premalignant lesions giving selective advantage to neoplastic cells (14). Recent studies have shown

Correspondence: S.D. Silva, Departamento de Cirurgia de Cabeça e Pescoço e Otorrinolaringologia, Hospital A.C. Camargo, Rua Prof. Antonio Prudente, 211, 01509-010 São Paulo, SP, Brasil. Fax: +55-5541-3341/0326. E-mail: sabrinadaniela@hotmail.com

Received November 21, 2011. Accepted August 15, 2012. Available online September 7, 2012. Published December 17, 2012. 
that knockout mice of p16 (INK4a) have more spontaneous tumors and increased cancer susceptibility when exposed to carcinogen agents than wild-type mice (2).

Despite the tremendous progress in surgical techniques, radiotherapy, and chemotherapy, the prognosis for patients with HNSCC has been only slightly improved during the past three decades (15). Chemotherapy has produced unsatisfactory results in head and neck tumors and novel approaches, including treatment tailoring by pharmacogenetic analysis and new molecular-targeted drugs, are required. Gene therapy is emerging as potentially efficacious techniques for many tumors. However, the scarcity of effective therapies may reflect the lack of knowledge about the influence of tumor-related molecular abnormalities on responsiveness to drugs and how it can impact on patient survival. Although some studies have examined the association between molecular markers in HNSCC, few have evaluated their effects on survival. Among those that have attempted to examine these effects, few examined the molecular markers adjusting for effects of clinical stage, lifestyle habits, and pathological characteristics. Thus, the prognostic significance remains to be addressed in patients with oropharynx and larynx squamous cell carcinoma. In this report, we showed the clinicopathological impact and prognostic implications of negative p16 (INK4a) expression in HNSCC patients followed-up for at least 10 years.

\section{Material and Methods}

\section{Study population}

A retrospective study was performed by analyzing 40 oropharynx and 35 larynx specimens from primary HNSCC patients (all histological diagnosis were confirmed) diagnosed and treated at the Department of Head and Neck Surgery and Otorhinolaryngology, A.C. Camargo Hospital, São Paulo, Brazil. The eligibility criteria included previously untreated patients with diagnosis of squamous cell carcinoma submitted to treatment only in this institution. Demographic (age, gender, and race), lifestyle (smoking habit and alcohol consumption), clinical (macroscopy, tumor site, and clinical stage), and pathological factors (histological grade, vascular embolization, perineural infiltration, lymphatic permeation, capsular rupture, and surgical margins) were analyzed. The tumors were staged according to the 2002 version of the International Union Against Cancer (TNM) classification and grouped as early (T1+T2) or advanced clinical stage (T3+T4). All cases were followed-up after treatment, with disease recurrence being confirmed microscopically. The histological grade was determined on the basis of the classification proposed by the World Health Organization (16). Vascular embolization and lymphatic permeation were classified according to the presence or absence of neoplastic cells, located both in the wall and lumen of the blood or lymphatic vessels, respectively; perineural infiltration was considered to be present when the tissue adjacent to the peri- and/or intra-tumoral nerves was involved by the neoplastic cells; surgical margins were considered to be involved when invasive and/or in situ carcinoma was observed in the margins of the surgical specimens. This study was carried out with approval of the Human Research Ethics Committee of Hospital A.C. Camargo.

\section{Tissue microarray (TMA) and immunohistochemistry}

To construct the TMA, core biopsies were taken from previously defined areas, with a Tissue Microarray apparatus (Beecher Instruments ${ }^{\circledR}$, USA). Tissue cores with $1.0 \mathrm{~mm}$ were punched from each specimen and arrayed in duplicate on a recipient paraffin block. Cores were spaced $0.2 \mathrm{~mm}$ apart. After cutting $(3 \mu \mathrm{m})$ on the recipient block and transferring with an adhesive tape to coated slides for subsequent UV cross-linkage (Instrumedics Inc. ${ }^{\circledR}$, USA), the slides were dipped in paraffin to prevent oxidation, and maintained in $\mathrm{a}-20^{\circ} \mathrm{C}$ freezer.

For immunostaining, the sections were deparaffinized and rehydrated in graded ethanol solutions, treated with peroxide to quench endogenous peroxidase $\left(0.3 \% \mathrm{H}_{2} \mathrm{O}_{2}\right.$ for $15 \mathrm{~min}$ ) and blocked for avidin/biotin (DAKO Biotin Blocking System ${ }^{\circledR}$, Dako Cytomation, Denmark) and for protein (DAKO ${ }^{\circledR}$ Protein Block Serum-Free, Dako Cytomation). Antigen recovery was performed by wet heating in a pressure cooker. Slides were incubated with anti-p16 (INK4a; Santa Cruz, USA, 1:200). The immunohistochemical reaction was carried out in duplicate at different TMA levels, representing 4-fold redundancy for each case. Positive and negative controls were included in all reactions. p16 (INK4a) was assessed by two kinds of negative controls: 1) omitting the primary antibody and incubating slides with phosphate-buffered saline; 2) replacing the primary antibody with normal mouse serum. After scanning each tumor specimen in a low-power field to choose the most stained area, at least five fields were evaluated under high power. The presence of a clearly visible dark brown precipitation was considered to be an immunoreaction. The cells were considered to be positive if they showed intense diffuse staining on the slides. Focal staining was scored as p16 (INK4a) negative. Evaluation of p16 (INK4a) included the nuclear proportion of reactive cells within the tumors and the staining intensity. The two pathologists who scored the immunostaining to determine the percentage and color intensities of stained cells were blind to the outcome and clinical aspects of each tumor specimen. Each core was scanned in low-power field to choose the most stained area predominant in at least $10 \%$ of tumor cells (17).

\section{Statistical analysis}

For frequency analysis of contingency tables, statistical analyses of associations between variables were performed by the chi-square test or the Fisher exact test (with significance set at $P<0.05)$. The overall survival (OS) was defined as the interval between the beginning of treatment 
and the date of death or the last information for censored observations. The disease-free interval (DFI) was measured from the date of treatment to the date when recurrence was diagnosed. Overall survival and disease-free survival probabilities were estimated by the Kaplan-Meier method, and the log-rank test was applied to assess the significance of differences among actuarial survival curves. For statistical analysis, the samples were categorized into two groups: negative versus positive reaction. All analyses were performed using the statistical software package STATA(STATA Corporation, USA).

\section{Results}

The study population consisted of 75 patients, 71 $(94.7 \%)$ males and $4(5.3 \%)$ females, with a mean age of 58 years (range 21-76 years). A history of alcohol consumption was observed in 62 (87.3\%) patients and tobacco smoking in $71(94.7 \%)$. With regard to the ethnic group, $67(89.3 \%)$ were Caucasians and $8(10.7 \%)$ non-Caucasians. The time of complaint was defined as the time between the date of recognition of the first sign or symptom of the disease by the patient and the date of first visit to a professional who was qualified to refer the patient for definitive diagnosis and treatment. The mean time of the complaint was 5 months (range 1-72 months). The oropharynx was the most frequent site (45 cases, $53.3 \%$ ), followed by the larynx (30 cases, $46.7 \%$ ). A total of 22 cases (33.3\%) were in an early clinical stage (T1+T2) and $44(66.7 \%)$ in an advanced clinical stage (T3+T4). Twenty-seven patients had metastatic cervical lymph nodes (40.9\%). Vascular embolization was detected in 57 of the 75 eligible cases (79.2\%), lymphatic permeation in $46(63.9 \%)$, perineural infiltration in $32(44.4 \%)$, and involved margins in $6(8.6 \%)$. Twenty-six cases $(34.7 \%)$ were histologically well differentiated (grade I), 42 cases (56\%) moderately differentiated or grade II and 6 cases (9.3\%) poorly differentiated or grade III.

The immunohistochemical results are summarized in Table 1. p16 (INK4a) positivity was nuclear and intracytoplasmic (Figure 1A). Forty cases (53.3\%) were positive for p16 (INK4a; Figure 1A) and this positivity was more intense in non-smoker patients $(P=0.050)$, presenting negative vascular embolization $(P=0.018)$, negative lymphatic permeation $(P=0.002)$, and clear surgical margins $(P=$ $0.050)$ (Table 1). Thirty-five cases were negative $(46.7 \%$; Figure 1B).

Twenty-nine $(39.7 \%)$ patients had tumor recurrence during the study within a mean time of 23.2 months (range 1-87.7 months). At the end of the follow-up period, 36 (48\%) patients were alive and $39(52 \%)$ had died (Table 1$)$. Thirtysix $(48 \%)$ patients died because of the HNSCC and $3(4 \%)$ of other causes during follow-up. OS time ranged from 1 to 126.13 months (mean: 55.5 months). The 5-year rates for OS and DFI were 56 and $64 \%$, respectively (Figure $2 \mathrm{~A}$ and B). Importantly, our data show that p16 (INK4a) im-
Table 1. Correlation between p16 (INK4a) protein expression and clinicopathological variables in head and neck squamous cell carcinomas.

\begin{tabular}{|c|c|c|}
\hline \multirow[t]{2}{*}{ Characteristics } & \multicolumn{2}{|c|}{ p16 (INK4a) [N (\%)] } \\
\hline & Negative & Positive \\
\hline \multicolumn{3}{|l|}{ Gender } \\
\hline Male & $33(46.5)$ & $38(53.5)$ \\
\hline Female & $2(50)$ & $2(50)$ \\
\hline \multicolumn{3}{|l|}{ Race } \\
\hline Caucasian & $32(47.8)$ & $35(52.2)$ \\
\hline Non-Caucasian & $3(37.5)$ & $5(62.5)$ \\
\hline \multicolumn{3}{|l|}{ Smoking habit } \\
\hline No & $0^{*}$ & $4(100)$ \\
\hline Yes & $35(49.3)$ & $36(50.7)$ \\
\hline \multicolumn{3}{|l|}{ Alcohol consumption } \\
\hline No & $6(46.2)$ & $7(53.8)$ \\
\hline Yes & $29(46.8)$ & $33(53.2)$ \\
\hline \multicolumn{3}{|l|}{ Tumor site } \\
\hline Pharynx & $30(47.6)$ & $33(52.4)$ \\
\hline Larynx & $5(41.7)$ & $7(58.3)$ \\
\hline \multicolumn{3}{|l|}{ T stage } \\
\hline $\mathrm{T} 1+\mathrm{T} 2$ & $8(36.4)$ & $14(63.6)$ \\
\hline $\mathrm{T} 3+\mathrm{T} 4$ & $23(52.3)$ & $21(47.7)$ \\
\hline \multicolumn{3}{|l|}{ Lymph nodes } \\
\hline NO & 19 (48.7) & $20(51.3)$ \\
\hline $\mathrm{N}+$ & $12(44.4)$ & $15(55.6)$ \\
\hline \multicolumn{3}{|l|}{ Histological grade } \\
\hline I & $14(53.8)$ & $12(46.2)$ \\
\hline II & $19(45.2)$ & $23(54.8)$ \\
\hline III & $2(28.6)$ & $5(71.4)$ \\
\hline \multicolumn{3}{|c|}{ Vascular embolization } \\
\hline No & $3(20)^{*}$ & $12(80)$ \\
\hline Yes & $31(54.4)$ & $26(45.6)$ \\
\hline \multicolumn{3}{|c|}{ Lymphatic permeation } \\
\hline No & $6(23.1)^{*}$ & $20(76.9)$ \\
\hline Yes & $28(60.9)$ & $18(39.1)$ \\
\hline \multicolumn{3}{|l|}{ Perineural infiltration } \\
\hline No & $21(52.5)$ & $19(47.5)$ \\
\hline Yes & $13(40.6)$ & $19(59.4)$ \\
\hline \multicolumn{3}{|l|}{ Capsular rupture } \\
\hline No & $23(52.3)$ & $21(47.7)$ \\
\hline Yes & $12(40)$ & $18(60)$ \\
\hline \multicolumn{3}{|l|}{ Surgical margins } \\
\hline Negative & $27(42.2)^{*}$ & $37(57.8)$ \\
\hline Positive & $5(83.3)$ & $1(16.7)$ \\
\hline \multicolumn{3}{|l|}{ Status } \\
\hline Alive & 14 (38.9) & $22(61.1)$ \\
\hline Dead & $21(53.8)$ & $18(46.2)$ \\
\hline
\end{tabular}

Data are reported as number with percent (considering cases with complete information) in parentheses. ${ }^{*} \mathrm{P}<0.05$ (chi-square or Fisher exact tests). 
munolabeling was able to predict the overall survival probability (log-rank test, $P=0.055$ ), since patients whose tumors showed positive intracytoplasmic p16 (INK4a) expression had a higher overall survival than patients with negative reactions (Figure $2 \mathrm{C}$ ). There was a significantly lower survival probability for patients with lymph node metastasis (log-rank test, $P=0.050$ ) (Figure 2D) and the presence of capsular rupture (log-rank test, $P=0.0010$ ) (data not shown). According to the primary site, patients with tumors in the oropharynx $(56.7 \%)$ had shorter 5 -year overall survival rates than patients with lesions in the larynx (47.4\%), although the OS curves did not differ significantly $(P=0.495)$. DFI ranged from 1 to 87 months (mean: 23.2 months). Association with a higher risk of recurrence was observed in tumors presenting capsular rupture (logrank test, $P=0.0083)$. In the present study, no association occurred between p16 (INK4a) expression and disease-free survival (log-rank test, $\mathrm{P}=0.0788$ ) (data not shown).

\section{Discussion}

Since its discovery as a CDKI in 1993, the tumor suppressor p16 (INK4a) has gained widespread importance in cancer research $(1,4,7-13,18,19)$. The frequent mutations and deletions of CDKN2A in human cancer cell lines first suggested an important role for $\mathrm{p} 16$ (INK4a) in carcinogenesis rivaled in frequency only by the p53 tumor suppressor gene (20). The present study provides further insight into the real clinicopathological impact and prognostic implications of p16 (INK4a) immunohistochemical expression in a particular set of 75 HNSCC patients followed up on a long-term basis for at least 10 years.

In human neoplasms, CDKN2A is silenced in at least three ways: homozygous deletion, methylation of the promoter, and point mutation. The first two mechanisms are responsible for the majority of inactivation events in most primary tumors (20). The mechanism of action of the CDKI p16 (INK4a) blocks the transcription of important cell-cycle regulatory proteins among them, the enzymes phosphorylating $\mathrm{pRb}$ and it results in cell-cycle arrest $(5,6)$. pRb inactivation is usually reciprocal with p16 (INK4a) expression in several tumors $(6,21,22)$. Even though the molecular basis for overexpression of p16 (INK4a) in HNSCC is unclear, some studies have evaluated the relationship between $\mathrm{p} 16$ (INK4a) and human papillomavirus (HPV), suggesting that the activation of $\mathrm{p} 16$ (INK4a) in cell-cycle regulatory cascade
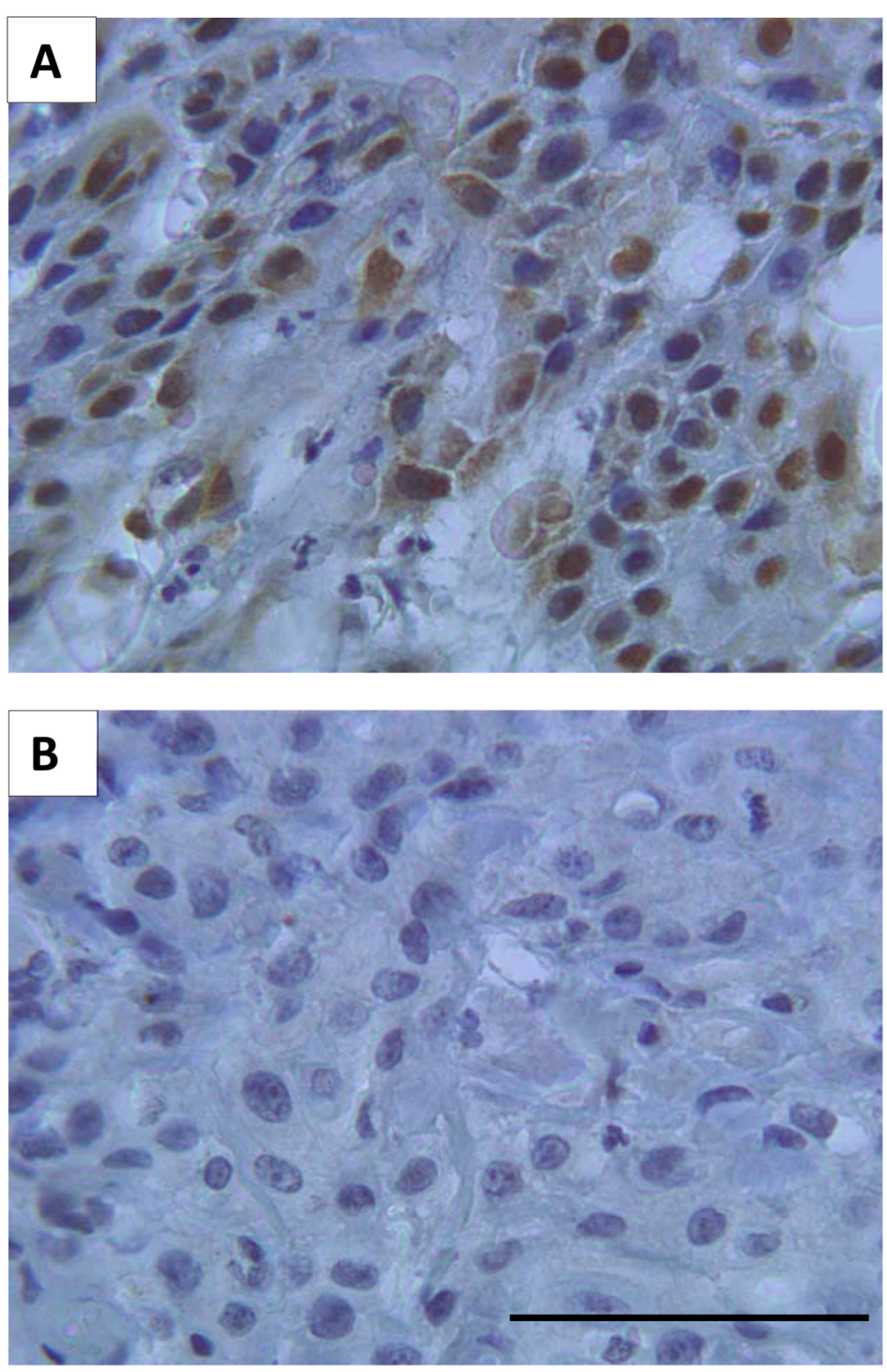

Figure 1. Representative immunohistochemical reactions for p16 (INK4a) in 75 head and neck squamous cell carcinoma (HNSCC) samples. Positive p16 (INK4a) reaction was found in $53.3 \%$ of the HNSCC $(A)$, whereas morphologically normal areas (spotted in the tumor microarray as control) and $46.7 \%$ of the tumors showed negative expression $(B)$. Bar $=100 \mu \mathrm{m}$ for both panels. may be linked with HPV status $(3,23-26)$. Recently, it has become increasingly clear that HPVs replicate by interfering with normal cell cycle control mechanisms. Evidence suggests that malignant transformation is intimately related to these processes, and that the oncogenic potential of papillomaviruses probably lies in their ability to alter cell cycle checkpoints, thereby leading to the accumulation and transmission of genetic abnormalities (27). Studies have shown that the E6 and E7 oncoproteins of high-risk HPVs, particularly HPV 16 and HPV 18, respectively bind 

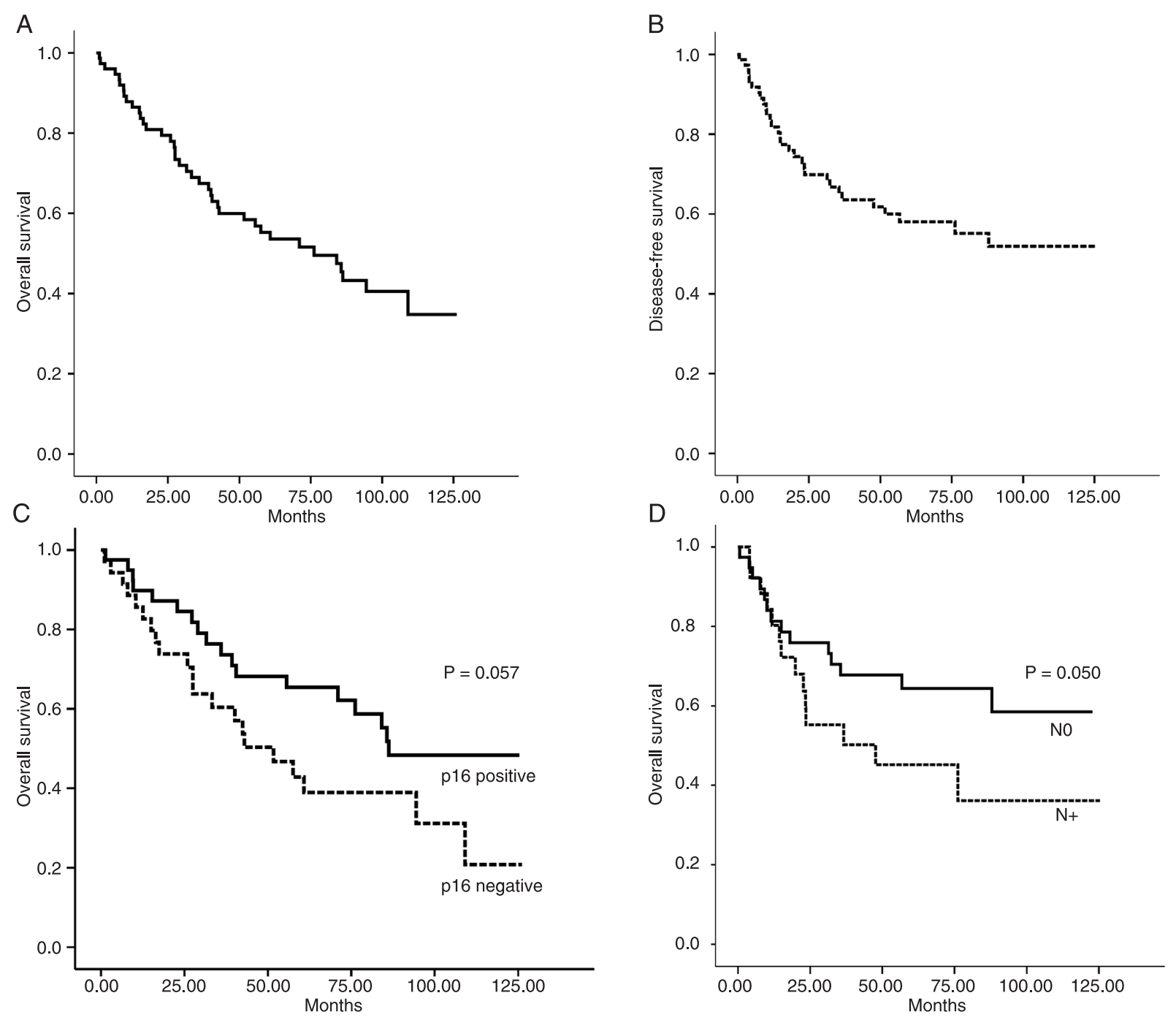

Figure 2. Overall survival analysis $(A)$ and disease-free survival $(B)$ of the 75 head and neck squamous cell carcinoma (HNSCC) samples studied. HNSCC cases with negative p16 (INK4a) immunolabeling had a shorter survival rate (C) compared to cases with positive staining $(\mathrm{P}<0.057$, log-rank test and Kaplan-Meier method). The overall survival probability $(D)$ was shorter for HNSCC patients with positive lymph nodes $(\mathrm{N}+; \mathrm{P}<0.050$, log-rank test and Kaplan-Meier method).

to the $\mathrm{p} 53$ and $\mathrm{pRb}$ tumor suppressor proteins, which are involved in the regulation of growth control $(27,28)$. However, this is a controversial issue, which remains subject to debate in HPV-related HNSCC because it depends on epidemiological factors as well as technical improvements in the detection of HPV (29).

Aclearer knowledge of alterations in p16 (INK4a) expression, as well as their real consequences would contribute to the understanding of the pathogenesis of HNSCC. Although p16 (INK4a) may be involved in cell senescence, the physiologic role of $\mathrm{p} 16$ (INK4a) is still uncertain. In the present study, we compared the protein expression and its relationship with the two major sites of HNSCC excluding the oral cavity, i.e., the larynx and oropharynx. Different anatomical sites have not been distinguished in the majority of studies involving HNSCC cases. However, the behavior of tumors arising at various sites differs significantly, suggesting different intrinsic tumor properties (30). Our analysis, in agreement with other HNSCC studies, did not show any statistically significant difference between immunohistochemical expression of p16 (INK4a) and tumor localization, with a similar distribution of positive immunostaining being observed at both sites ( 52.4 and $58.3 \%$ in the oropharynx and larynx, respectively).

The development and progression of HNSCC have been shown to be dependent on various cellular genetic 
and epigenetic events, especially alterations of the cell cycle machinery at various checkpoints. Among our samples, the tumors presenting positive vascular embolization ( $P$ $=0.018)$, positive lymphatic permeation $(P=0.002)$, and positive surgical margins $(P=0.050)$ showed an inverse correlation with p16 (INK4a) expression. Hence, p16 (INK4a)-negative status was associated with more aggressive pathological conditions, probably because this protein is critical for the regulation of cell proliferation, and the inactivation of this gene is crucial for the tumorigenesis process (27). Furthermore, insights into the role of p16 (INK4a) in cancer have come from the genetic analysis of precancerous lesions and various tissue culture models. It is now believed that loss of $\mathrm{p} 16$ is an early and often critical event in tumor progression. Consequently, p16 (INK4a) is a major tumor suppressor gene whose frequent loss occurs early in many human cancers (14). Interestingly, p16 (INK4a) expression was more intense and significantly correlated with non-smoker patients $(P=0.050)$. Kong et al. (28) showed that in tobacco-related HNSCC, p16 (INK4a) is often absent because of homologous deletion or promoter hypermethylation.

Regarding survival probability, the 5-year rate for HNSCC patients was $48 \%$, which may be explained by the fact that in most cases the tumor was diagnosed in advanced clinical stages. In agreement with most literature data, our results of p16 (INK4a) expression were associated with lower overall survival probability (log-rank test, $\mathrm{P}=$ 0.055 ), suggesting that patients with negative $\mathrm{p} 16$ (INK4a) protein expression are at a higher risk of death $(3,13)$. To further improve survival rates, a clearer understanding of the carcinogenic mechanisms involved is important, thereby allowing the identification of patients at high risk of developing tumor recurrences or dying. Similarly, a significant association was observed with lower survival probability and the presence of lymph node metastasis (log-rank test, $\mathrm{P}=$

\section{References}

1. Orlow I, Lacombe L, Hannon GJ, Serrano M, Pellicer I, Dalbagni $\mathrm{G}$, et al. Deletion of the p16 and p15 genes in human bladder tumors. J Natl Cancer Inst 1995; 87: 1524-1529.

2. Quelle DE, Ashmun RA, Hannon GJ, Rehberger PA, Trono $\mathrm{D}$, Richter $\mathrm{KH}$, et al. Cloning and characterization of murine p16INK4a and p15INK4b genes. Oncogene 1995; 11: 635645.

3. Smith EM, Rubenstein LM, Hoffman H, Haugen TH, Turek LP. Human papillomavirus, p16 and p53 expression associated with survival of head and neck cancer. Infect Agent Cancer 2010; 5: 4.

4. Takeuchi S, Bartram CR, Seriu T, Miller CW, Tobler A, Janssen JW, et al. Analysis of a family of cyclin-dependent kinase inhibitors: p15/MTS2/INK4B, p16/MTS1/INK4A, and p18 genes in acute lymphoblastic leukemia of childhood. Blood 1995; 86: 755-760.

5. Poznic M. Retinoblastoma protein: a central processing unit.
0.0050 ) and capsular rupture (log-rank test, $P=0.0010)$. In addition, the presence of capsular rupture was associated with a higher risk of tumor recurrence (log-rank test, $\mathrm{P}=$ 0.0083). The metastatic dissemination of HNSCC usually occurs through the lymphatic system (31). For these tumors, metastasis to regional lymph nodes is the strongest predictor and the most important prognostic factor, which leads to a reduction in the probability of disease control and shorter survival of the patients $(31,32)$. There is a critical necessity to understand the molecular mechanisms in HNSCC patients. Based on the present outcomes, future studies should perhaps focus on the upstream events that lead to p16 (INK4a) expression and its mechanism of regulation, looking for better therapeutic strategies that can improve the clinical course of HNSCC patients because the death rate remains extremely high. Gene therapy represents a potential opportunity to advance the management of HNSCC patients and improve prognosis.

Our findings suggest that a genetic approach may eventually help improve the ability to predict prognosis and to prevent the tumor progression of HNSCC patients that should enter surveillance programs. Finally, analysis of the results obtained in our selected clinical population followed for almost a decade showed that it is able to predict prognosis on the basis of p16 (INK4a), given that its negative expression is important for determining the parameters associated with tumor progression and poor outcomes in HNSCC.

\section{Acknowledgments}

The authors would like to acknowledge Carlos Ferreira Nascimento and Severino Ferreira for technical assistance. Research supported by FAPESP (\#06/61039-8 and CEPID/FAPESP (\#98/14335). S.D. Silva is the recipient of a FAPESP fellowship (\#06/61040-6).
J Biosci 2009; 34: 305-312.

6. Sano T, Oyama T, Kashiwabara K, Fukuda T, Nakajima T. Expression status of $\mathrm{p} 16$ protein is associated with human papillomavirus oncogenic potential in cervical and genital lesions. Am J Pathol 1998; 153: 1741-1748.

7. Takeuchi S, Seriu T, van Dongen JJ, Szczepanski T, Tsukasaki K, Takeuchi N, et al. Allelotype analysis in relapsed childhood acute lymphoblastic leukemia. Oncogene 2003; 22: 6970-6976.

8. Rother J, Jones D. Molecular markers of tumor progression in melanoma. Curr Genomics 2009; 10: 231-239.

9. Sanchez-Fayos Calabuig P, Martin Relloso MJ, Porres Cubero JC. [Genetic profile and molecular bases of pancreatic carcinogenesis]. Gastroenterol Hepatol 2007; 30: 592-596.

10. Salam I, Hussain S, Mir MM, Dar NA, Abdullah S, Siddiqi MA, et al. Aberrant promoter methylation and reduced expression of p16 gene in esophageal squamous cell carcinoma from 
Kashmir valley: a high-risk area. Mol Cell Biochem 2009; 332: 51-58.

11. Ye Y, Wang D, Su C, Rong T, Guo A. Combined detection of p53, p16, Rb, and EGFR mutations in lung cancer by suspension microarray. Genet Mol Res 2009; 8: 1509-1518.

12. Attaleb M, El hamadani W, Khyatti M, Benbacer L, Benchekroun N, Benider A, et al. Status of p16(INK4a) and E-cadherin gene promoter methylation in Moroccan patients with cervical carcinoma. Oncol Res 2009; 18: 185-192.

13. Koscielny S, Dahse R, Ernst G, von Eggeling F. The prognostic relevance of p16 inactivation in head and neck cancer. ORL J Otorhinolaryngol Relat Spec 2007; 69: 30-36.

14. Rocco JW, Sidransky D. p16(MTS-1/CDKN2/INK4a) in cancer progression. Exp Cell Res 2001; 264: 42-55.

15. Zhou X, Temam S, Oh M, Pungpravat N, Huang BL, Mao $\mathrm{L}$, et al. Global expression-based classification of lymph node metastasis and extracapsular spread of oral tongue squamous cell carcinoma. Neoplasia 2006; 8: 925-932.

16. Wahi P, Cohen B, Luthra U, Torloni H. Histological typing of oral and oropharyngeal tumours. Geneva: World Health Organization; 1971.

17. Kononen J, Bubendorf L, Kallioniemi A, Barlund M, Schraml $\mathrm{P}$, Leighton $\mathrm{S}$, et al. Tissue microarrays for high-throughput molecular profiling of tumor specimens. Nat Med 1998; 4: 844-847.

18. Sinha P, Bahadur S, Thakar A, Matta A, Macha M, Ralhan $\mathrm{R}$, et al. Significance of promoter hypermethylation of $\mathrm{p} 16$ gene for margin assessment in carcinoma tongue. Head Neck 2009; 31: 1423-1430.

19. Takeuchi S, Bartram CR, Wada M, Reiter A, Hatta Y, Seriu $\mathrm{T}$, et al. Allelotype analysis of childhood acute lymphoblastic leukemia. Cancer Res 1995; 55: 5377-5382.

20. Liggett WH Jr, Sidransky D. Role of the p16 tumor suppressor gene in cancer. J Clin Oncol 1998; 16: 1197-1206.

21. Li AF, Li AC, Tsay SH, Li WY, Liang WY, Chen JY. Alterations in the p16INK4a/cyclin D1/RB pathway in gastrointestinal tract endocrine tumors. Am J Clin Pathol 2008; 130: 535542.

22. Richmond-Sinclair NM, Lee E, Cummings MC, Williamson $\mathrm{R}$, Muller K, Green AC, et al. Histologic and epidemiologic correlates of P-MAPK, Brn-2, pRb, p53, and p16 immunostaining in cutaneous melanomas. Melanoma Res 2008; 18 :
336-345.

23. Hoffmann M, Ihloff AS, Gorogh T, Weise JB, Fazel A, Krams $\mathrm{M}$, et al. p16(INK4a) overexpression predicts translational active human papillomavirus infection in tonsillar cancer. Int J Cancer 2010; 127: 1595-1602.

24. Nichols AC, Faquin WC, Westra WH, Mroz EA, Begum S, Clark JR, et al. HPV-16 infection predicts treatment outcome in oropharyngeal squamous cell carcinoma. Otolaryngol Head Neck Surg 2009; 140: 228-234.

25. Nakao Y, Yang X, Yokoyama M, Ferenczy A, Tang SC, Pater $\mathrm{MM}$, et al. Induction of p16 during immortalization by HPV 16 and 18 and not during malignant transformation. Br J Cancer 1997; 75: 1410-1416.

26. Singhi AD, Westra WH. Comparison of human papillomavirus in situ hybridization and p16 immunohistochemistry in the detection of human papillomavirus-associated head and neck cancer based on a prospective clinical experience. Cancer 2010; 116: 2166-2173.

27. Kim YT, Zhao M. Aberrant cell cycle regulation in cervical carcinoma. Yonsei Med J 2005; 46: 597-613.

28. Kong CS, Narasimhan B, Cao H, Kwok S, Erickson JP, Koong A, et al. The relationship between human papillomavirus status and other molecular prognostic markers in head and neck squamous cell carcinomas. Int J Radiat Oncol Biol Phys 2009; 74: 553-561.

29. Lim KP, Hamid S, Lau SH, Teo SH, Cheong SC. HPV infection and the alterations of the pRB pathway in oral carcinogenesis. Oncol Rep 2007; 17: 1321-1326.

30. dos Reis PP, Poli-Frederico RC, dos Santos RM, Nishimoto IN, Kowalski LP, Rogatto SR. Distinct regions of loss of heterozygosity on $22 q$ in different sites of head and neck squamous cell carcinomas. Med Sci Monit 2002; 8: BR89BR94.

31. Kowalski LP, Bagietto R, Lara JR, Santos RL, Silva JF Jr, Magrin J. Prognostic significance of the distribution of neck node metastasis from oral carcinoma. Head Neck 2000; 22: 207-214.

32. Fletcher AM, Heaford AC, Trask DK. Detection of metastatic head and neck squamous cell carcinoma using the relative expression of tissue-specific mir-205. Trans/ Oncol 2008; 1 : 202-208. 